\title{
GENERALIZATION OF BATEMAN POLYNOMIALS
}

\author{
ASAD ALI*, MUHAMMAD ZAFAR IQBAL, BILAL ANWER, ATHER MEHMOOD \\ Department of Mathematics and Statistics, University of Agriculture Faisalabad, Pakistan
}

*Corresponding author:: mrasadali5100@gmail.com

\begin{abstract}
In this paper, generalize the Bateman polynomials in terms of generalized hypergeometric function of the type ${ }_{p} F_{p}$. Establish different forms of extended polynomials such as series expansion, generating functions and recurrence relations.
\end{abstract}

\section{INTRODUCTION}

Bateman polynomials are the family of $F_{n}$ orthogonal polynomials. Many of researchers generalized the classical results on the Bateman polynomials. A large dedicated literature, numbers of relevant properties, extensions, generalizations and applications of Bateman polynomials are available in [1], [2], [4], [7], [10] and [11]. The Bateman polynomials $f_{n}(x)$ generated by

$$
\sum_{n=0}^{\infty} f_{n}(x) t^{n}=(1-t)^{-1} \psi\left(\frac{-4 x t}{(1-t)^{2}}\right)
$$

have the following classical properties.

$$
\begin{gathered}
f_{n}(x)=\sum_{k=0}^{\infty} \frac{(-n)_{k}(1+n)_{k} \gamma_{k} x^{k}}{\left(\frac{1}{2}\right)_{k}(1)_{k}}, \\
f_{n}(x)={ }_{2} F_{2}(-n, 1+n ; 1,1 ; x), \\
x f_{n}^{\prime}(x)-n f_{n}(x)=-n f_{n-1}(x)-x f_{n-1}^{\prime}(x), n \geq 1,
\end{gathered}
$$

Received 2019-04-08; accepted 2019-05-13; published 2019-09-02.

2010 Mathematics Subject Classification. 26C05, 65Q30.

Key words and phrases. Bateman polynomials; generating functions; recurrence relations.

(C)2019 Authors retain the copyrights of their papers, and all open access articles are distributed under the terms of the Creative Commons Attribution License. 


$$
\begin{aligned}
& x f_{n}^{\prime}(x)-n f_{n}(x)=-\sum_{k=0}^{n-1} f_{k}(x)-2 x \sum_{k=0}^{n-1} f_{k}^{\prime}(x), n \geq 1 \\
& x f_{n}^{\prime}(x)-n f_{n}(x)=\sum_{k=0}^{n-1}(-1)^{n-k}(1+2 k) f_{k}(x), n \geq 1 .
\end{aligned}
$$

\section{MAIN RESULTS}

In this section we determine generalized properties of classical Bateman polynomials, series expansion, generating function and recurrence relations. For this let $\psi(u)$ have a formal power- series expansion

$$
\psi(u)=\sum_{n=0}^{\infty} \gamma_{n} u^{n}
$$

Define a polynomials $f_{n}^{(\alpha)}(x)$ by

$$
\sum_{n=0}^{\infty} f_{n}^{(\alpha)}(x)^{n}=(1-t)^{-1-\alpha} \psi\left(\frac{-p^{p} x t}{q^{q}(1-t)^{p}}\right)
$$

where $p \geq 2, q=p-1$ and $\alpha$ is any non-negative real parameter.

Theorem 2.1. If $n$ is non-negative integer then,

$$
f_{n}^{(\alpha)}(x)=\frac{(1+\alpha)_{n}}{n !} \sum_{k=0}^{n} \frac{(-n)_{k}\left(\frac{1+\alpha+n}{q}\right)_{k}\left(\frac{2+\alpha+n}{q}\right)_{k} \ldots\left(\frac{q+\alpha+n}{q}\right)_{k} x^{k} \gamma_{k}}{\left(\frac{1+\alpha}{p}\right)_{k}\left(\frac{2+\alpha}{p}\right)_{k} \ldots .\left(\frac{p+\alpha}{p}\right)_{k}}
$$

Proof: From (2.1) and (2.2)

$$
\sum_{n=0}^{\infty} f_{n}^{(\alpha)}(x) t^{n}=\sum_{k=0}^{\infty} \frac{(-1)^{k}(p)^{p k} x^{k} t^{k} \gamma_{k}}{(q)^{q k}(1-t)^{1+\alpha+p k}}
$$

By using (1), pp 58 of [1]

$$
\sum_{n=0}^{\infty} f_{n}^{(\alpha)}(x) t^{n}=\sum_{n=0}^{\infty} \sum_{k=0}^{\infty} \frac{(-1)^{k}(p)^{p k}(1+\alpha)_{n+p k} \gamma_{k} x^{k} t^{n+k}}{(q)^{q k} n !(1+\alpha)_{p k}}
$$

By using Lemma 11, pp 57 of [1]

$$
\sum_{n=0}^{\infty} f_{n}^{(\alpha)}(x) t^{n}=\sum_{n=0}^{\infty} \sum_{k=0}^{n} \frac{(-1)^{k}(p)^{p k}(x)^{k}(1+\alpha)_{n+q k} \gamma_{k} t^{n}}{(q)^{q k}(n-k) !(1+\alpha)_{p k}}
$$




$$
=\sum_{n=0}^{\infty} \sum_{k=0}^{n} \frac{(1+\alpha)_{n}}{n !} \frac{(-1)^{k} n !(p)^{p k}(x)^{k}(1+\alpha+n)_{q k} \gamma_{k} t^{n}}{(q)^{q k}(n-k) !(1+\alpha)_{p k}}
$$

equating the coefficients of $t^{n}$, we obtain (2.3).

Theorem 2.2. If $n \geq 1$, then

$$
f_{n}^{(\alpha)}(x)=\frac{(1+\alpha)_{n}}{n !}{ }_{p} F_{p}(-n, \frac{1+\alpha+n}{q}, \frac{2+\alpha+n}{q} \ldots \frac{q+\alpha+n}{q} ; \underbrace{1,1 \ldots 1}_{p-\text { times }} ; x) .
$$

Proof: If we choose

$$
\gamma_{k}=\frac{\left(\frac{1+\alpha}{p}\right)_{k}\left(\frac{2+\alpha}{p}\right)_{k} \ldots .\left(\frac{p+\alpha}{p}\right)_{k}}{(k !)^{p+1}} .
$$

in (2.3) then our yield is (2.4).

Theorem 2.3. If $n$ is non-negative integer then,

$$
x f_{n}^{\prime(\alpha)}(x)-n f_{n}^{(\alpha)}(x)=-(\alpha+n) f_{n-1}^{(\alpha)}(x)-q x f_{n-1}^{\prime(\alpha)}(x) .
$$

\section{Proof:}

In order to derive (2.5), consider

$$
\begin{gathered}
F=\sum_{n=0}^{\infty} f_{n}^{(\alpha)}(x) t^{n}=(1-t)^{-1-\alpha} \psi(v) \\
\text { where, } v=\frac{-p^{p} x t}{q^{q}(1-t)^{p}}
\end{gathered}
$$

Differentiate with respect to $x$

$$
F_{x}=\sum_{n=0}^{\infty} f_{n}^{\prime(\alpha)}(x) t^{n}=(1-t)^{-1-\alpha} \psi^{\prime}(v) \frac{-p^{p} t}{q^{q}(1-t)^{p}},
$$


Differentiate with respect to $t$

$$
\begin{gathered}
F_{t}=\sum_{n=0}^{\infty} f_{n}^{(\alpha)}(x) n t^{n-1}=(1+\alpha)(1-t)^{-2-\alpha} \psi(v)-(1-t)^{-1-\alpha} \psi^{\prime}(v) \frac{\partial v}{\partial t}, \\
\text { where, } \frac{\partial v}{\partial t}=\frac{-p^{p} x(1+q t)}{q^{q}(1-t)^{p+1}} . \\
F_{t}=\sum_{n=0}^{\infty} f_{n}^{(\alpha)}(x) n t^{n-1}=(1+\alpha)(1-t)^{-2-\alpha} \psi(v)-x \frac{p^{p}(1-t)^{-2-\alpha-p}(1+q t)}{q^{q}} \psi^{\prime}(v),
\end{gathered}
$$

Therefore $F$ satisfies the partial differential equation

$$
\begin{gathered}
x(1+q t) F_{x}-t(1-t) F_{t}+(1+\alpha) t F=0 . \\
x(1+q t) \sum_{n=0}^{\infty} f_{n}^{\prime(\alpha)}(x) t^{n}-t\left(1-t \sum_{n=0}^{\infty} f_{n}^{(\alpha)}(x) n t^{n-1}\right)+(1+\alpha) t \sum_{n=0}^{\infty} f_{n}^{(\alpha)}(x) t^{n}=0, \\
\sum_{n=0}^{\infty}\left[x f_{n}^{\prime(\alpha)}(x)-n f_{n}^{(\alpha)}(x)\right] t^{n}=-\sum_{n=0}^{\infty}(1+\alpha+n) f_{n}^{(\alpha)}(x) t^{n+1}-q x \sum_{n=0}^{\infty} f_{n}^{\prime(\alpha)}(x) t^{n+1}, \\
=-\sum_{n=1}^{\infty}(\alpha+n) f_{n-1}^{(\alpha)}(x) t^{n}-q x \sum_{n=1}^{\infty} f_{n-1}^{\prime}(x) t^{n},
\end{gathered}
$$

which leads to (2.5).

Theorem 2.4. If $n$ is non-negative integer then,

$$
x f_{n}^{\prime(\alpha)}(x)-n f_{n}^{(\alpha)}(x)=-(1+\alpha) \sum_{k=0}^{n-1} f_{k}^{(\alpha)}(x)-p x \sum_{k=0}^{n-1} f_{k}^{\prime(\alpha)}(x) .
$$

\section{Proof:}


$F$ also satisfies the partial differential equation

$$
\begin{gathered}
x F_{x}-x t F_{x}+p x t F_{x}-t F_{t}+t^{2} F_{t}+(1+\alpha) t F=0 . \\
x F_{x}-t F_{t}=-\frac{(1+\alpha) t}{1-t} F-\frac{p x t}{1-t} F_{x} . \\
x \sum_{n=0}^{\infty} f_{n}^{\prime}(\alpha)(x) t^{n}-t \sum_{n=0}^{\infty} f_{n}^{(\alpha)}(x) n t^{n-1}=-(1+\alpha) \sum_{n=0}^{\infty} t^{n+1} \sum_{k=0}^{\infty} f_{k}^{(\alpha)}(x) t^{k}-p x \sum_{n=0}^{\infty} t^{n+1} \sum_{k=0}^{\infty} f_{k}^{\prime(\alpha)}(x) t^{k}, \\
\sum_{n=0}^{\infty}\left[x f_{n}^{\prime}(\alpha)(x)-n f_{n}^{(\alpha)}(x)\right] t^{n}=-(1+\alpha) \sum_{n=0}^{\infty} \sum_{k=0}^{\infty} f_{k}^{(\alpha)}(x) t^{n+k+1}-p x \sum_{n=0}^{\infty} \sum_{k=0}^{\infty} f_{k}^{\prime(\alpha)}(x) t^{n+k+1} \\
=-\sum_{n=1}^{\infty}\left[(1+\alpha) \sum_{k=0}^{n-1} f_{k}^{(\alpha)}(x)-p x \sum_{k=0}^{n-1} f_{k}^{\prime}(\alpha)(x)\right] t^{n},
\end{gathered}
$$

which leads to (2.6).

Theorem 2.5. If $n$ is non-negative integer then,

$$
x f_{n}^{\prime(\alpha)}(x)-n f_{n}^{(\alpha)}(x)=\sum_{k=0}^{n-1}(-q)^{n-k}(1+\alpha+p k) f_{k}^{(\alpha)}(x)
$$

\section{Proof:}

$F$ satisfies the partial differential equation

$$
\begin{gathered}
x F_{x}+q x t F_{x}-t F_{t}-q t^{2} F_{t}+p t^{2} F_{t}+(1+\alpha) t F=0 . \\
x F_{x}-t F_{t}=-\frac{(1+\alpha) t}{1+q t} F-\frac{p t^{2}}{1+q t} F_{t} \\
\sum_{n=0}^{\infty}\left[x f_{n}^{\prime(\alpha)}(x)-n f_{n}^{(\alpha)}(x)\right] t^{n}=-(1+\alpha) \sum_{n=0}^{\infty} \sum_{k=0}^{\infty}(-q)^{n} f_{k}^{(\alpha)}(x) t^{n+k+1}-p \sum_{n=0}^{\infty} \sum_{k=0}^{\infty}(-q)^{n} f_{k}^{(\alpha)}(x) k t^{n+k+1},
\end{gathered}
$$




$$
=\sum_{n=1}^{\infty} \sum_{k=0}^{n-1}(-q)^{n-k}(1+\alpha+p k) f_{k}^{(\alpha)}(x) t^{n}
$$

which gives $(2.7)$.

For $\alpha=0$ and $p=2$ the equations (2.2) to (2.7) reduces to (1.1) to (1.6).

Theorem 2.6. If $n \geq 1$, then the polynomials $f_{n}^{(\alpha)}(x)$ also satisfying the following property

$$
\sum_{n=0}^{\infty} f_{n}^{(\alpha)}(x) t^{n}=(1-t)^{-1-\alpha}{ }_{p} F_{p}(\frac{1+\alpha+n}{p}, \frac{2+\alpha+n}{p} \ldots \frac{q+\alpha+n}{p} ; \underbrace{1, \ldots 1}_{p-\text { times }} ; \frac{-p^{p} x t}{q^{q}}) .
$$

\section{Acknowledgments}

The authors express their sincere gratitude to Dr. Ghulam Farid for useful discussions and invaluable advice.

\section{REFERENCES}

[1] E. D. Rainville, Special Functions, The Macmillan Company, New York, 1960.

[2] G. Andrews, R. Askey and R. Roy, Special Functions, Cambridge University Press, 2004.

[3] G. Andrews, R. Askey and R. Roy. Special Functions, Cambridge University Press, 1999.

[4] S. B. Trickovic and M. S. Stankovic, On the orthogonality of classical orthogonal polynomials, Integral Transforms Spec. Funct., 14(2003), 129-138.

[5] R. Diaz and E. Pariguan, On hypergeometric functions and Pochhammer k-symbol, Divulg. Mat., 15 (2007), $179-192$.

[6] K. Y. Chen and H. M. Srivastava, A limit relationship between Laguerre and Hermite polynomials. Integral Transforms Spec. Funct., 16(2005), 75 - 80.

[7] E. H. Doha, H. M. Ahmed and S. I. El-Soubhy, Explicit formulae for the coefficients of integrated expansions of Laguerre and Hermite polynomials and their integrals, Integral Transforms Spec. Funct., 20 (2009), 491 - 503.

[8] I. Krasikov and A. Zarkh, Equioscillatory property of the Laguerre polynomials, J. Approx. Theory, 162(2010), 2021 2047.

[9] S. Alam and A. K. Chongdar, On generating functions of modified Laguerre polynomials, Rev. Real Academia de Ciencias. Zaragoza, 62(2007), $91-98$.

[10] M. A. Khan and A. K. Shukla, On some generalized Sister Celines polynomials, Czechoslovak Math. J., 49(3) (1999), 527-545.

[11] H. M. Srivastava and P. W. Karlsson, Multiple Gaussian Hypergeometric Series, HalstedPress (Ellis Horwood Limited, Chichester), John Wiley and Sons, New York, Chichester, Brisbane and Toronto, 1985. 